Supporting Information

\title{
Branched Supramolecular Copolymers: Inducing Branching in Bisurea-Based Monomers Using
}

\section{Multi-Sulfonate Molecules}

\author{
Ishay Columbus, $, *, \uparrow$ Noga Eren, ${ }^{a}$ Renana Elitsur, ${ }^{a}$ \\ Maya Davidovich-Pinhas, ${ }^{b, *}$ and Roy Shenhar ${ }^{a, *}$
}

${ }^{a}$ Institute of Chemistry and the Center for Nanoscience and Nanotechnology, The Hebrew University of Jerusalem, Jerusalem 9190401, Israel.

${ }^{b}$ Faculty of Biotechnology and Food Engineering, Technion - Israel Institute of Technology, Haifa, 32000, Israel 

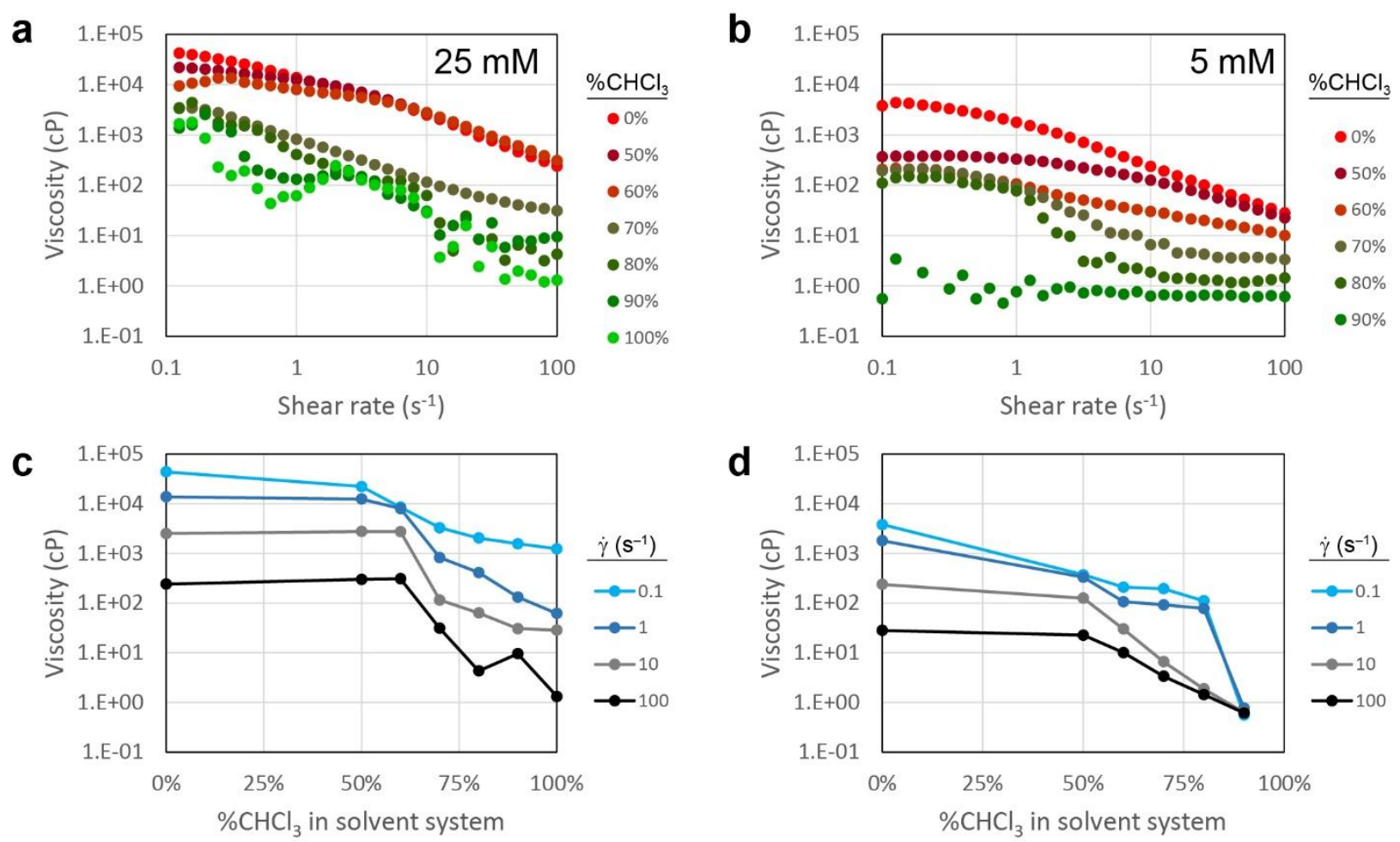

Figure S1. $(\mathrm{a}, \mathrm{b})$ Shear rate-dependent viscosity measurements of pure EHUT in $\mathrm{CHCl}_{3} /$ toluene at different solvent compositions (denoted by the volume percent of chloroform) and two different EHUT concentrations. (c,d) Viscosity data at different shear rates taken from the corresponding plots in $(a, b)$, showing that the transition from tubular-related to filamentous-related viscosity behavior occurs at a solvent composition of $\sim 60-65 \% \mathrm{CHCl}_{3}$. 


\section{Experiment $\mathrm{A}$}
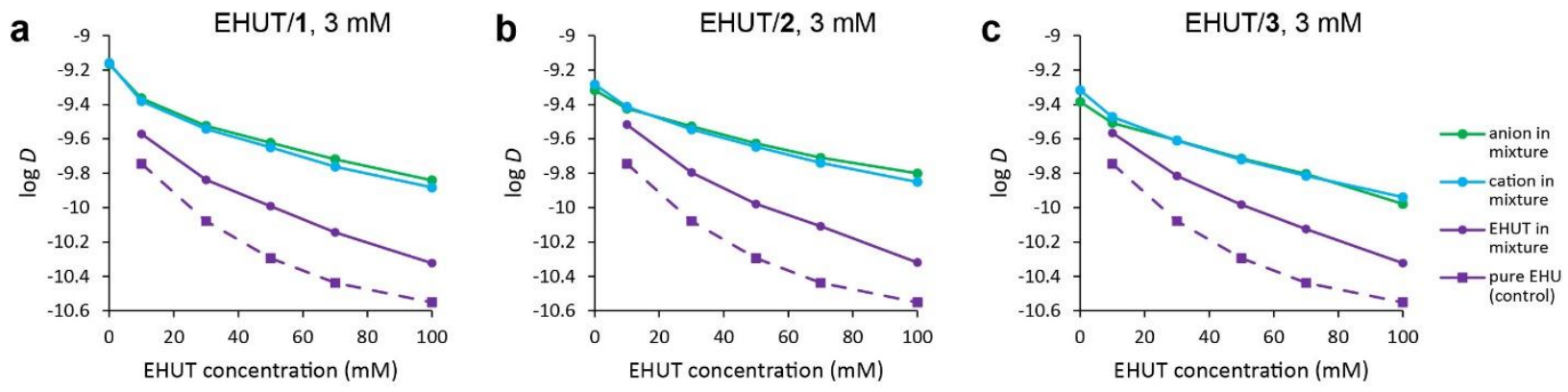

Experiment B
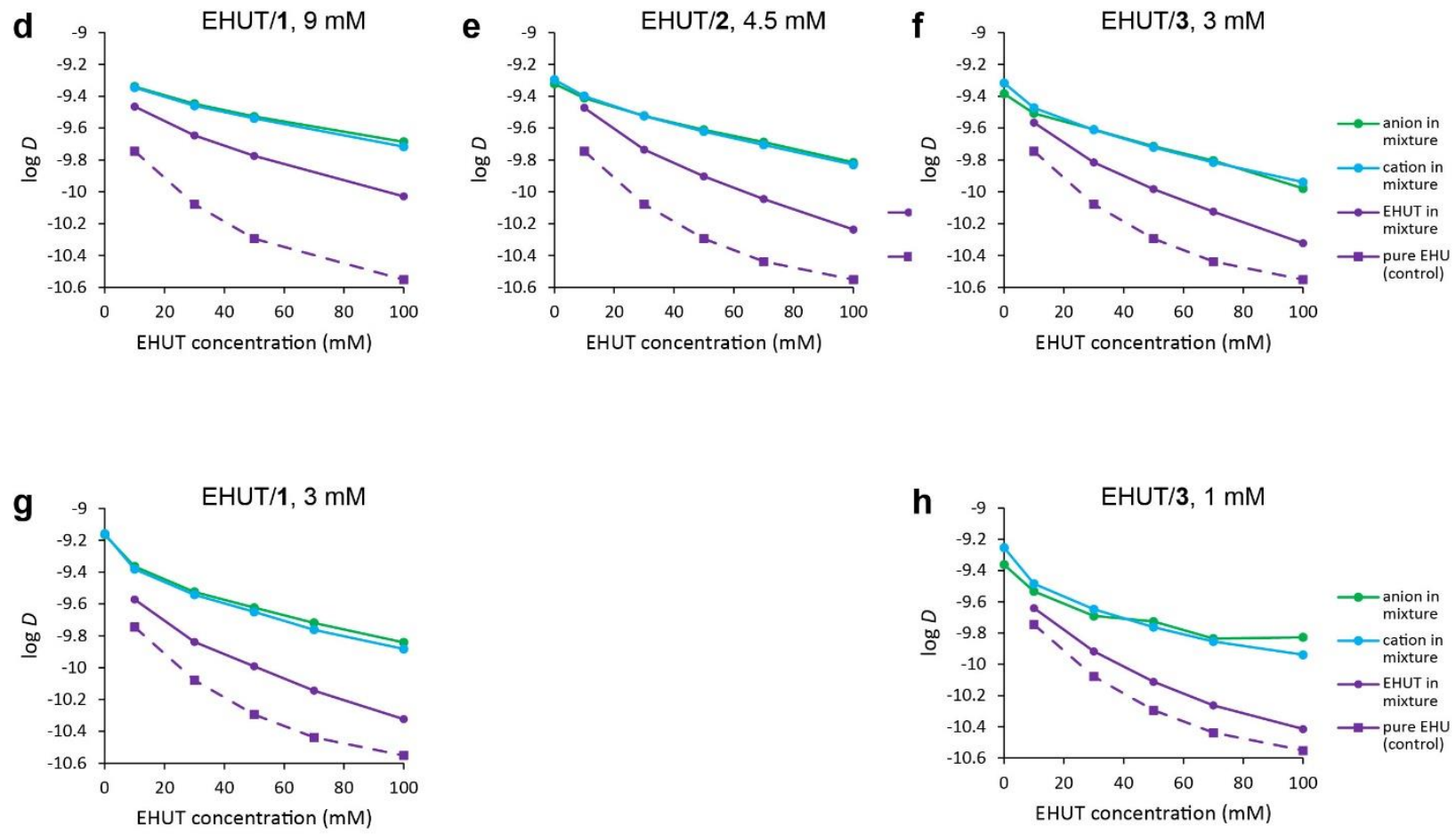

Figure S2. Full DOSY data sets of all systems with different MSU concentrations in 1:1 $\mathrm{CDCl}_{3} /$ toluene- $d_{8}$. Top row (panels a-c): comparison at constant MSU concentration (3 mM; "Experiment A"). Middle row (panels d-f): comparison at constant sulfonate group concentration (9 mM; "Experiment B"). Bottom row (panels g,h): another comparison at constant sulfonate group concentration $(3 \mathrm{mM})$. The curves of the diffusion rates of pure EHUT (dashed line) in the same solvent system are given for reference. Panels f,g were replicated from panels c,a (respectively) to facilitate visual comparison. 


\section{General implication of high association constants on branching in supramolecular polymers}

Substituting unity for the value of $p_{\mathrm{B}}$ and applying equation 2 to equation 5 leads to a simplified expression for the average degree of polymerization:

$$
N \approx \frac{f / r+2}{2-f p_{\mathrm{S}}}
$$

This equation is structurally analogous to the equation derived by Carothers for gelation, and shows that for supramolecular polymers featuring high association constants the average degree of polymerization in branched systems depends on the fraction of interacting functionality that causes the branching $(p s)$ and the system composition $(r)$ as the main variables. The lack of dependence on the fraction of interacting EHUT units $\left(p_{\mathrm{A}}\right)$ is justified by the chain stopping effect, which is pronounced for large association constants, and removes the dependence of the degree of polymerization not only the on the concentration of the supramolecular monomer but also on the association constant (which is expressed by $p_{\mathrm{A}}$ in equation 5). ${ }^{1}$ Additionally, this equation predicts that a supramolecular gel would form when $p_{\mathrm{S}}$ approaches $2 / f$ (for $f \geq 2$ ). This is obviously an overestimate (because gelation depends on the largest polymer, not on the average degree of polymerization), but could serve as a useful guideline for gelation in branched supramolecular system.

\section{Role of water in the supramolecular EHUT/MSU assemblies}

Vlassopoulos et al. have shown that humidity reduces the viscosity of EHUT in dodecane. ${ }^{2}$ Similarly, Meijer and co-workers have shown that water molecules alter the structure of supramolecular polymers in alkanes. ${ }^{3}$ A certain amount of water is present in chloroform and toluene solutions ( 0.056 and $0.033 \mathrm{wt} \%$, respectively), and the MSU compounds might be hygroscopic as well, which raises a concern regarding the influence of residual water on the EHUT/MSU supramolecular assemblies formed. Yet, there are a few reasons to believe that 
besides a certain constant offset to all measured values, the effect observed should be attributed to the presence of the MSU and not to the influence of water molecules. First, the hydration levels determined by Karl Fischer titrations measured $<1 \mathrm{wt} \%$ for all compounds (EHUT and MSUs), and were not correlated the MSU valency. Additionally, we calculate that these levels of hydration contribute between $0.2-7.7 \%$ to the amount of water already present in the solvents. Therefore, any effect that might have been caused by the presence of water molecules did not arise indirectly by the introduction of water by the added MSUs but by the water present in the solvent system used. Considering that most of the experiments were performed at constant MSU concentrations (usually $3 \mathrm{mM}$ ), the water content should be considered nearly constant in all solutions. Thus, while the presence of water might have offset the diffusion constant and viscosity values measured, this effect should be constant to all solutions that were compared and the trends observed must be attributed to the presence of the MSU and not to the presence of residual water.

Second, anions are known to be stronger hydrogen bond acceptors than neutral molecules by 13 orders of magnitude owing to the concentration of charge density on specific atoms. ${ }^{4-6}$ Therefore, while the presence of water molecules may inlay some defects in the supramolecular assembly, this interference should be considered secondary to the effect of the MSUs.

Lastly, we also consider the possibility that water could serve as a bridging molecule between a sulfonate functionality and the oxygen atom of the urea group. While this possibility could not be dismissed, it is expected to be less favorable over direct hydrogen bonding between the sulfonate and urea moieties both for entropic reasons (a trimer is less favored than a dimer) as well as for enthalpic reasons (two hydrogen bonds between an anion and urea should be stronger than two hydrogen bonds in which one links two neutral species). Regardless, however, it is important to note that our theoretical model provides the same predictions even in the case that sulfonate 
interaction with EHUT occurs through the oxygen atoms of the urea groups mediated by a bridging water molecule. Implementing into the model the assumption that the MSU could interact not only through both the $\mathrm{NH}$ NH but also through the oxygen atoms of the urea groups leads to a similar dependence for equation 5 , where $p_{\mathrm{A}}$ is substituted with the term $\frac{\# A-\mathrm{B} \text { intractions }}{N_{\mathrm{A}}}$ (instead of $\frac{\# \text { interacting } \mathrm{A}}{N_{\mathrm{A}}}$ used in equation 3 to estimate the number of linkages). Counting all possible interactions between $\mathrm{A}, \mathrm{B}$, and $\mathrm{S}$, and using the assumption that the introduction of the MSUs only trades EHUT=EHUT interactions $(\mathrm{A} \cdots \mathrm{B})$ with sulfonate-EHUT interactions $(\mathrm{S} \cdots \mathrm{B}$, and in the current scenario also $\mathrm{S} \cdots \mathrm{A}$ ) leads to the same evaluation of $\frac{\# \mathrm{~A}-\mathrm{B} \text { intractions }}{N_{\mathrm{A}}}$ as $p_{\mathrm{A}}$ in the original model (i.e., using $p_{\text {в }}$ determined for pure EHUT and applying equation 2). Thus, the modified equation 5 produces the exact same values in both models. A corollary of the above discussion is that our conclusion on the induced branching by MSU 2 holds regardless of the type of interaction between the sulfonate functionalities and EHUT.

\section{Calculation of branching factor $g$ values}

Zimm and Stockmayer ${ }^{7}$ have extended the statistics of chain configuration to account for branching. In their theoretical approach, they define the ratio between the mean square radii of a branched and a linear chain of the same degree of polymerization as $g$. For units of valency 1 and 2 (i.e., chain stoppers and co-monomers), the value of $g$ is unity; for higher valencies and increasing number of branch points the values of $g$ decrease below unity, reflecting the compactness of branched molecules. Table I in their article summarizes the values of $g$ for branched chains as a function of branch unit valency and number of branch points, assuming random distribution of chain lengths between adjacent branch points. Figure $\mathbf{S 3}$ shows a secondorder polynomial fit of Zimm-Stockmayer values calculated for trivalent branch points for discrete 
number of branch points. This fit enables interpolation to obtain the average $g$ value for an average number of branch points per chain, $m$.

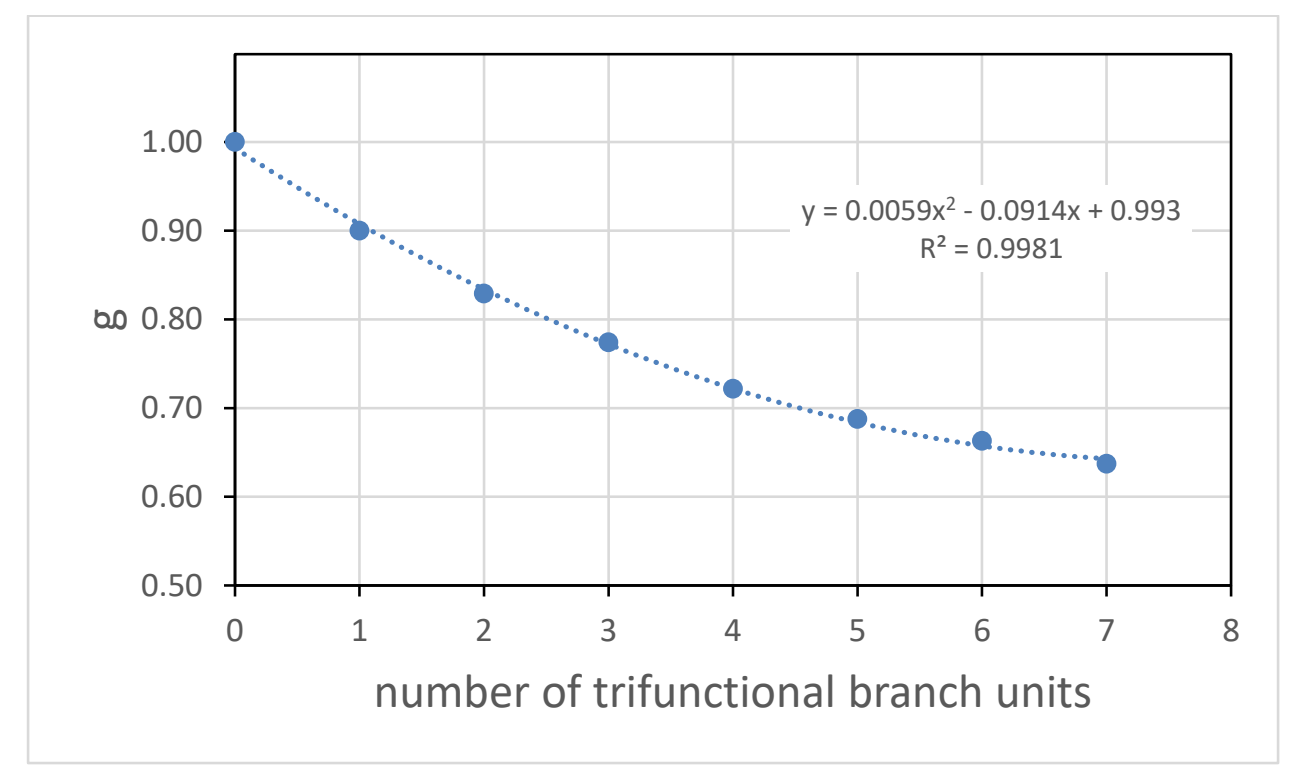

Figure S3. Quadratic polynomial fitting to Zimm-Stockmayer values of the branching factor $g$. 
Table S1. Calculated fractions of interacting MSUs ( $p_{\mathrm{MSU}}$ ) for all systems at $3 \mathrm{mM} \mathrm{MSU}$ concentration (Experiment A) in different solvents (calculated from DOSY data according to equation 6).

\begin{tabular}{|l|l|l|l|l|l|l|}
\hline & \multicolumn{3}{|c|}{$p_{\mathrm{MSU}}\left(\mathrm{CDCl}_{3} / \text { toluene- }_{8}\right)^{a}$} & \multicolumn{3}{c|}{$p_{\text {MSU }}\left(\text { toluene- } d_{8}\right)^{b}$} \\
\hline$c_{\text {EHUT }}$ & EHUT/1 & EHUT/2 & EHUT/3 & EHUT/1 & EHUT/2 & EHUT/3 \\
\hline 10 & 0.61 & 0.59 & 0.72 & 0.89 & 0.82 & 0.91 \\
\hline 20 & - & - & - & 0.92 & 0.88 & 0.91 \\
\hline 30 & 0.71 & 0.58 & 0.64 & 0.93 & 0.87 & 0.90 \\
\hline 40 & - & - & - & $0.93^{a}$ & $0.92^{b}$ & $0.94^{b}$ \\
\hline 50 & 0.77 & 0.65 & 0.71 & 0.93 & $0.93^{b}$ & - \\
\hline 70 & 0.81 & 0.71 & 0.76 & - & - & - \\
\hline 100 & 0.85 & 0.75 & 0.84 & - & - & - \\
\hline
\end{tabular}

${ }^{a}$ Calculated from interpolated value for the $D_{\text {anion. }}{ }^{b}$ Calculated using the corresponding $D_{\text {cation }}$ values instead of the $D_{\text {anion }}$ values (which were less reliable), relying on the overlap between the DOSY curves of the anion and cation.

Note that the fraction of interacting MSU molecules, which is determined directly from the experimental data, is rather independent on the type of MSU. This suggests that multiply interacting MSUs of type 2 and $\mathbf{3}$ come at the expense of singly interacting units (i.e., units that act as chains stoppers). It follows that the fraction of shortened linear EHUT chains in EHUT/2 and EHUT/3 should actually be lower than in EHUT/1 despite the larger number of sulfonate functionalities in MSUs 2 and 3. This lends further support to the conclusion that the reduced viscosities in EHUT/2 and EHUT/3 compared to EHUT/1 should be attributed to branching and not to an increased probability of forming shortened, linear chains. 


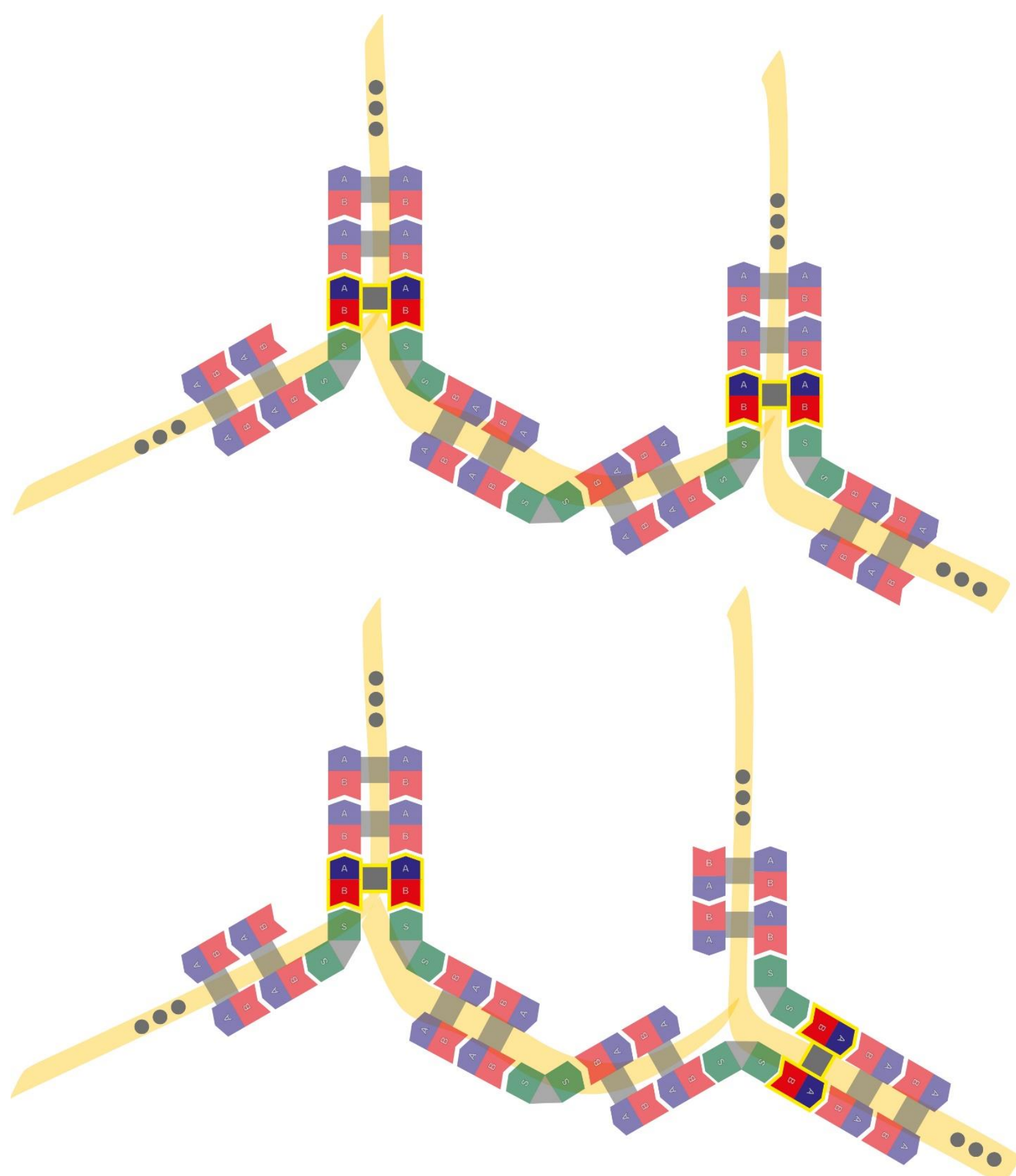

Figure S4. A schematic showing two possible arrangements of supramolecular chains that include two branch points induced by MSU 2 using a combination of parallel and anti-parallel arrangements of the EHUT units. 


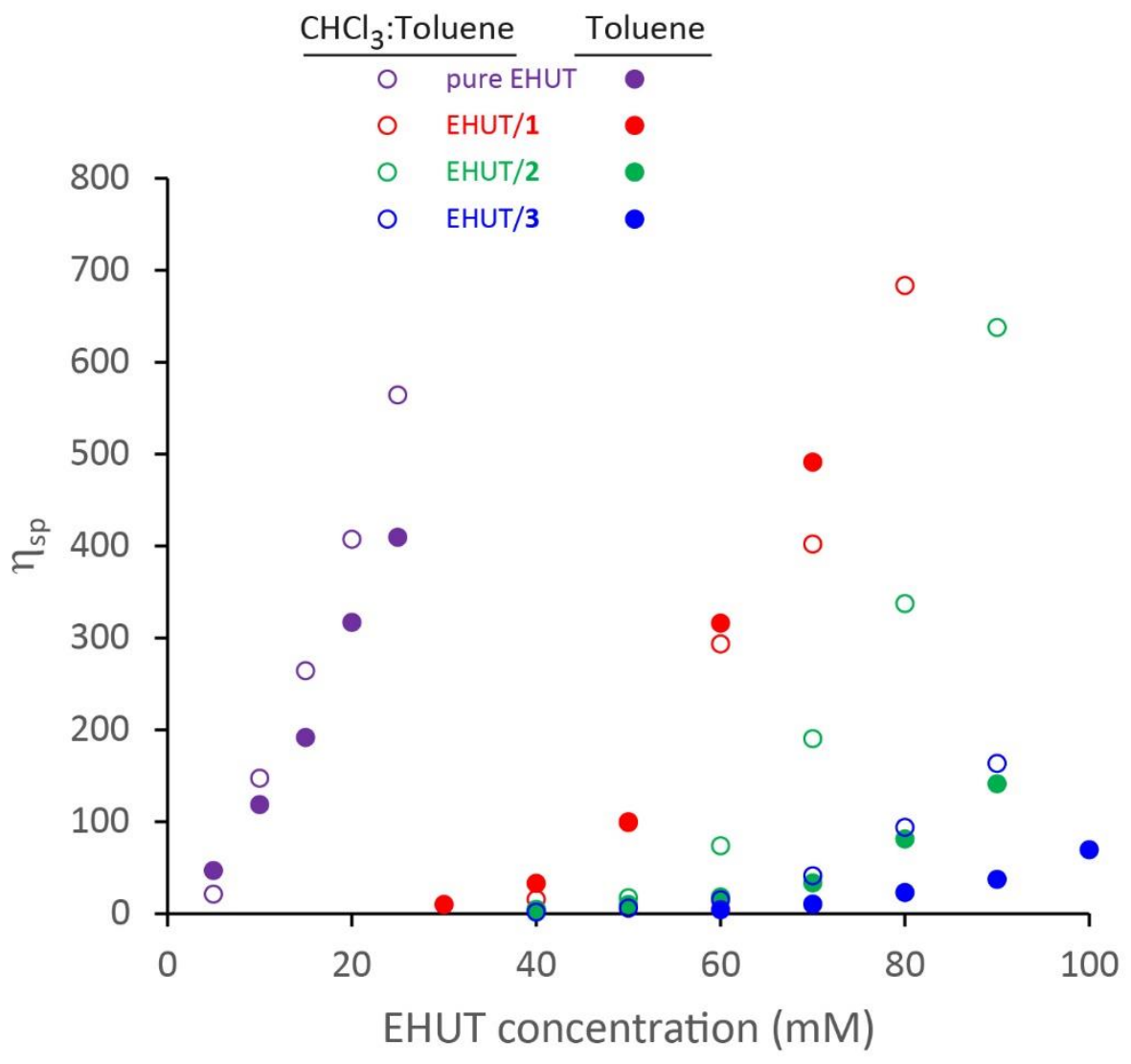

Figure S5. Comparison between the specific viscosities of all systems ( $3 \mathrm{mM}$ MSU concentration) in 1:1 $\mathrm{CHCl}_{3} /$ toluene (open symbols) vs. toluene (filled symbols). All measurements were acquired at shear rate of $100 \mathrm{~s}^{-1}$. 


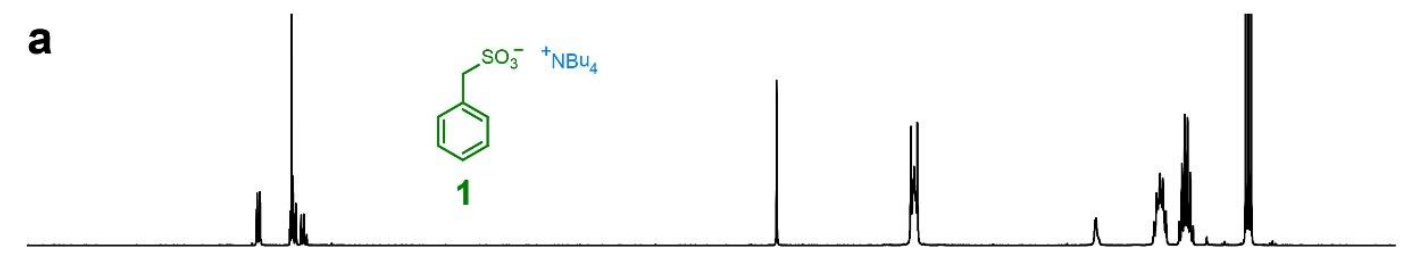

b
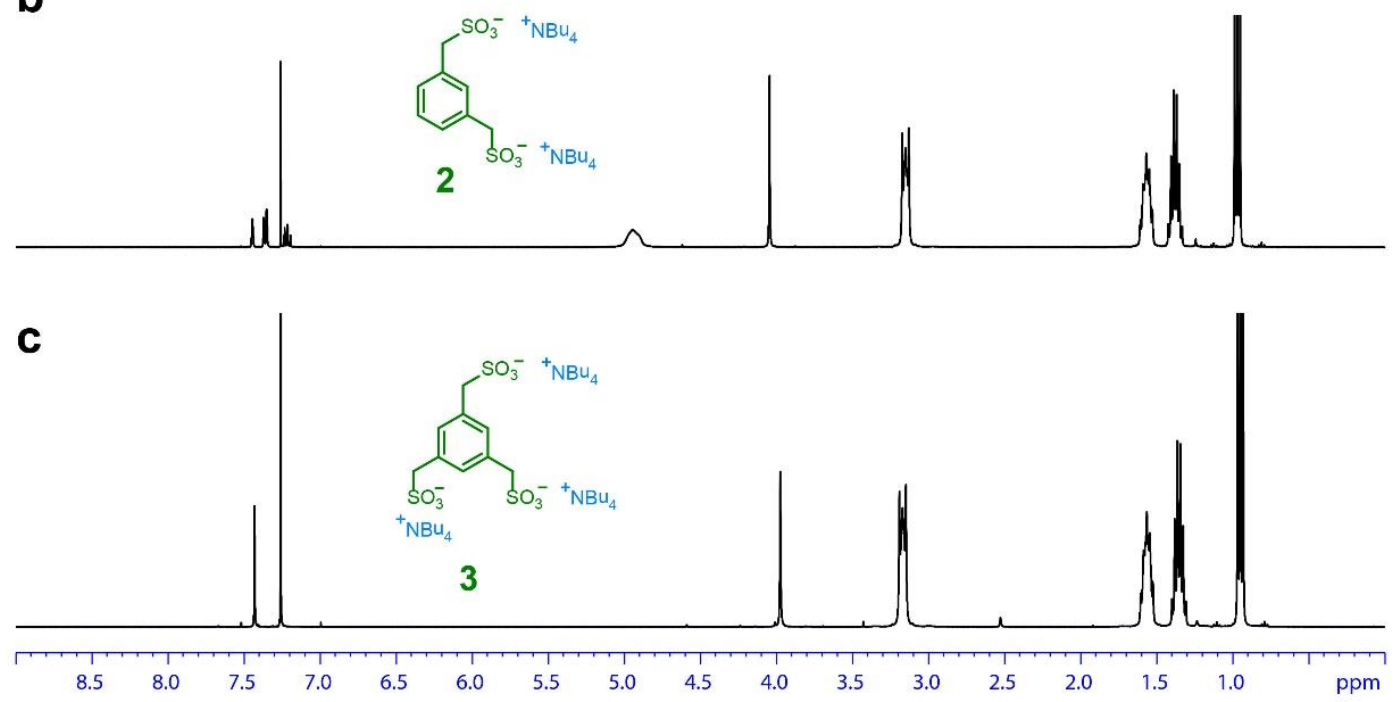

Figure S6. ${ }^{1} \mathrm{H}-\mathrm{NMR}$ spectra of MSUs 1-3 in $\mathrm{CDCl}_{3}$. 


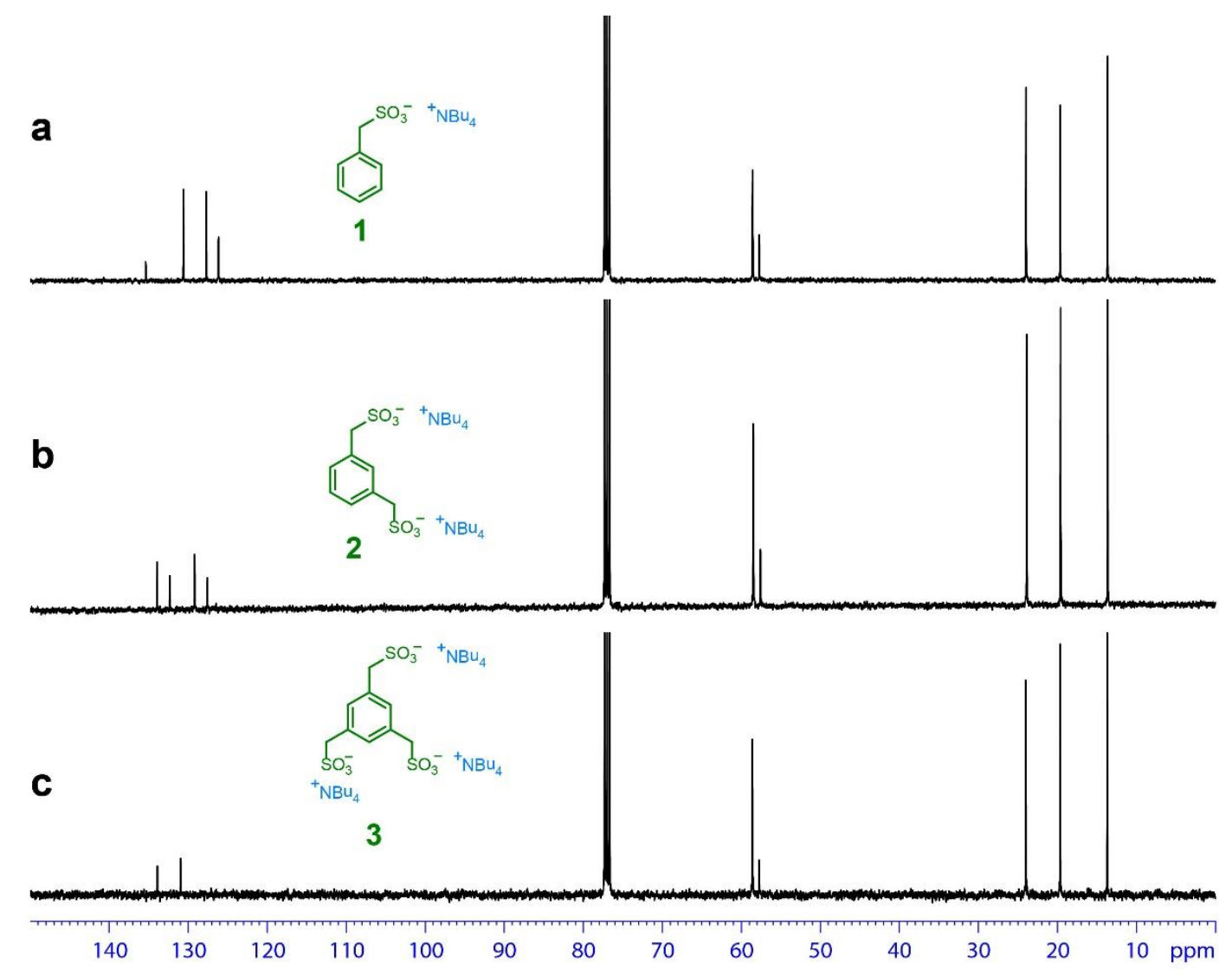

Figure S7. ${ }^{13} \mathrm{C}-\mathrm{NMR}$ spectra of MSUs $\mathbf{1 - 3}$ in $\mathrm{CDCl}_{3}$. 


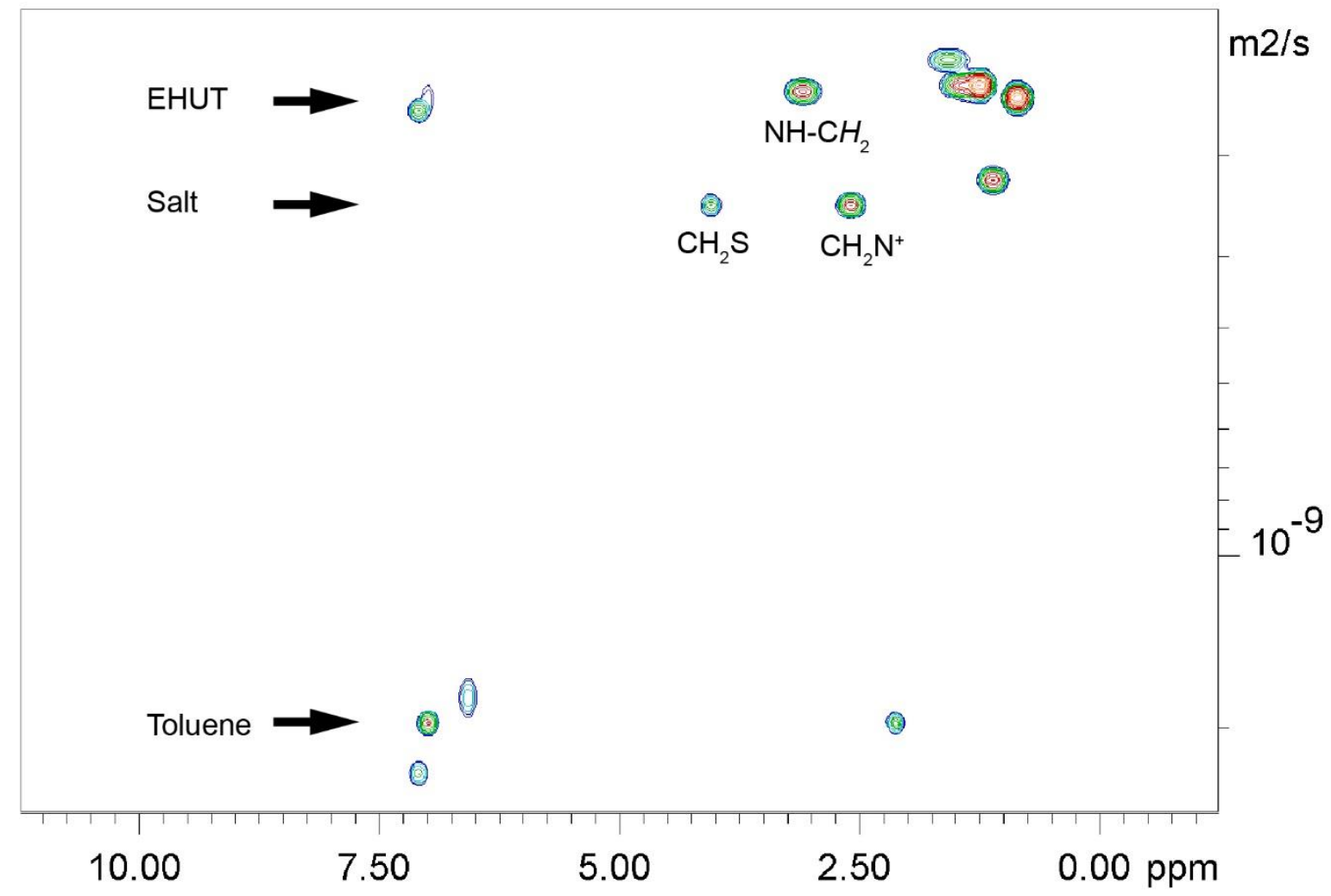

Figure S8. DOSY NMR spectrum of EHUT/3 (30:3 mM) in 1:1 $\mathrm{CDCl}_{3} /$ toluene- $d 8$.

\section{References}

1. Knoben, W.; Besseling, N. A. M.; Stuart, M. A. C. Chain Stoppers in Reversible Supramolecular Polymer Solutions Studied by Static and Dynamic Light Scattering and Osmometry. Macromolecules 2006, 39, 2643-2653.

2. Louhichi, A.; Jacob, A. R.; Bouteiller, L.; Vlassopoulos, D. Humidity Affects the Viscoelastic Properties of Supramolecular Living Polymers. J. Rheol. 2017, 61, 1173-1182.

3. Van Zee, N. J.; Adelizzi, B.; Mabesoone, M. F. J.; Meng, X.; Aloi, A.; Zha, R. H.; Lutz, M.; Filot, I. A. W.; Palmans, A. R. A.; Meijer, E. W. Potential Enthalpic Energy of Water in Oils Exploited to Control Supramolecular Structure. Nature 2018, 558, 100-103.

4. $\quad$ Pinault, T.; Cannizzo, C.; Andrioletti, B.; Ducouret, G.; Lequeux, F.; Bouteiller, L. Anions as Efficient Chain Stoppers for Hydrogen-Bonded Supramolecular Polymers. Langmuir 2009, 25, 8404-8407.

5. Boal, A. K.; Rotello, V. M. Redox-Modulated Recognition of Flavin by Functionalized Gold Nanoparticles. J. Am. Chem. Soc. 1999, 121, 4914-4915.

6. Pike, S. J.; Hutchinson, J. J.; Hunter, C. A. H-Bond Acceptor Parameters for Anions. J. Am. Chem. Soc. 2017, 139, 6700-6706.

7. Zimm, B. H.; Stockmayer, W. H. The Dimensions of Chain Molecules Containing Branches and Rings. J. Chem. Phys. 1949, 17, 1301-1314. 